Phase-field modelling of rapid solidification in alloy systems: Spontaneous grain refinement effects

This content has been downloaded from IOPscience. Please scroll down to see the full text. 2012 IOP Conf. Ser.: Mater. Sci. Eng. 33012109

(http://iopscience.iop.org/1757-899X/33/1/012109)

View the table of contents for this issue, or go to the journal homepage for more

Download details:

IP Address: 129.11.77.203

This content was downloaded on 17/02/2015 at $12: 48$

Please note that terms and conditions apply. 


\title{
Phase-field modelling of rapid solidification in alloy systems: Spontaneous grain refinement effects
}

\author{
A M Mullis \\ Institute for Materials Research, University of Leeds, Leeds, LS2-9JT, UK. \\ E-mail: A.M.Mullis@leeds.ac.uk
}

\begin{abstract}
Phase-field modelling of rapid alloy solidification, in which the rejection of latent heat from the growing solid cannot be ignored, has lagged significantly behind the modelling of conventional casting practises which can be approximated as isothermal. This is in large part due to the fact that if realistic materials properties are adopted the ratio of the thermal to solute diffusivity (the Lewis number) is typically $10^{3}-10^{4}$, leading to severe multi-scale problems. However, use of state-of-the-art numerical techniques such as local mesh adaptivity, implicit time-stepping and a non-linear multi-grid solver allow these difficulties to be overcome. Here we describe how the application of this model, formulated in the thin-interface limit, can help to explain the long-standing phenomenon of spontaneous grain refinement in deeply undercooled melts. We find that at intermediate undercoolings the operating point parameter, $\sigma^{*}$, may collapse to zero, resulting in the growth of non-dendritic morphologies such as doublons and 'dendritic seaweed'. Further increases in undercooling then lead to the re-establishment of stable dendritic growth. We postulate that remelting of such seaweed structures gives rise to the low undercooling instance of grain refinement observed in alloys.
\end{abstract}

\section{Introduction}

Dendritic solidification is a subject of enduring interest within the scientific community, both because dendrites are a prime example of spontaneous pattern formation and due to their pervasive influence on the engineering properties of metals. One long-standing problem with regard to the dendritic solidification of metals has been that of spontaneous grain refinement in undercooled pure melts, first reported to occur in Ni by Walker [1] in 1959. At a well defined undercooling, $\Delta T^{*}=140-150 \mathrm{~K}$, Walker observed an abrupt transition from a coarse columnar grain structure to a fine equiaxed structure, with a reduction in grain size of at least one order of magnitude. Similar behaviour was found in Co, with a value for $\Delta T^{*}$ of $\approx 180 \mathrm{~K}$. This effect has subsequently been identified in other pure metals $[2,3,4]$ and in a range of alloy systems $[5,6,7,8,9,10,11,12]$, in which a more complex evolutionary sequence is often observed as the undercooling is increased. At low undercooling an initially columnar growth pattern is observed which gives way to an equiaxed grain structure as the undercooling is increased beyond a critical value, $\Delta T_{1}^{*}$. At yet higher undercooling a second region of columnar growth is observed which, in most systems, is replaced by a second region of equiaxed growth, the critical undercooling for this second grain refinement transition being $\Delta T_{2}^{*}$.

In many of the systems in which spontaneous grain refinement is observed simultaneous measurement of the dendrite growth velocity has also been undertaken. As first demonstrated by Willnecker et al. [2] in levitation melted $\mathrm{Ni}$, such measurements reveal an apparent correlation between the velocity-undercooling relationship and grain refinement. Below $\Delta T^{*}$, growth velocity can be adequately represented by current dendrite growth models, with $V \propto \Delta T^{\beta}, \beta>1$. Above $\Delta T^{*}$ the 
velocity-undercooling relationship is approximately linear. In alloy systems this transition is usually observed to occur coincident with the $\Delta T_{2}^{*}$ transition. In most cases the transition to a grain refined microstructure at the lower undercooling, $\Delta T_{1}^{*}$, does not appear to have any obvious signature in the velocity undercooling curve. Moreover, differences between the as-solidified microstructures of the two grain refined regions indicate that there may be subtle differences in the underlying grain refinement mechanism. In the grain refined structures found at high undercooling the solute segregation pattern is spheroidal about a small dendrite fragment, whereas in grain refined materials formed at low undercooling some of the grains appear to contain small, equiaxed structures [13].

The origins of the effect are controversial. Early theories suggested a range of mechanisms which included nucleation ahead of the solidification front induced by the pressure pulse associated with solidification [1], recrystallisation, either during or immediately after solidification [14, 15], or the role of minor solute additions $[16,17,18]$. However, more recent theories regarding the origin of this phenomenon tend to invoke fragmentation of the primary crystal, either during [19,20] or immediately after $[21,22]$ recalescence. We have argued previously that the occurrence of a discontinuity in the velocity-undercooling curve $[2,23]$ coincident with the onset of grain refinement would tend to suggest the former of these possibilities is more likely, as would an apparent change in the morphology of the growth front revealed by high-speed imaging [24]. Specifically, we have suggested that a tip-splitting instability at the dendrite tip leads to the growth of an unstable 'dendritic seaweed' structure, which subsequently remelts to give the observed grain-refinement. Phase-field modelling of the thermally controlled solidification of a pure material at high undercooling [25] appear to support this suggestion, as does the observation, in deeply undercooled ultra-high purity $\mathrm{Cu}$, of a 'frozen in' seaweed morphology [26] above some critical undercooling. Moreover, above this critical undercooling a discontinuity in the velocity-undercooling curve was observed identical in character to that found in materials that display spontaneous grain refinement.

However, the dendritic fragmentation model of Schwarz et al. [21, 22] is perhaps still the most widely accepted model for spontaneous grain refinement. The model postulates that two characteristic timescales can be defined for dendritic growth from an undercooled melt. The first is the breakup time, $\tau_{\text {bu }}$, which is the time required for fragmentation of the side branches due to remelting and Rayleigh instability and is a monotonic function of the dendrite trunk radius, with small radii giving short breakup times. If, as seems likely from observations of dendritic growth in transparent systems such as xenon [27], dendrites are self-similar when scaled by the tip radius, $\rho$, this is equivalent to $\tau_{\text {bu }}$ being a monotonic function of $\rho$. The second timescale is the plateau time, $\tau_{\mathrm{pl}}$, which is the time the melt remains at, or around, the melting temperature during recalescence and is determined by the macroscopic heat extraction rate. The theory postulates that grain refinement occurs when $\tau_{\mathrm{bu}}<\tau_{\mathrm{pl}}$, which corresponds to the tip radius being below some critical value, $\rho^{*}$, determined by the heat extraction rate. Consequently, if it is assumed that $\rho$ varies with undercooling as predicted by marginal stability theory $[28,29]$ the model appears to offer a natural explanation for there being a single grain refinement transition in pure materials and two transitions in alloy systems (see Fig. 1).

The dependence of the dendrite tip radius upon undercooling as predicted by marginal stability theory for alloy systems, with its characteristic local minimum followed by a local maximum, has very much been a cornerstone of rapid solidification theory for the past 20 years. However, since the advent of microscopic solvability theory $[30,31]$ there can be considered to be no theoretical basis for marginal stability theory and the experimental evidence in support of the existence of either a local minimum, or a local maximum, in the tip radius is scant. Transparent analogue casting alloys, such as succinonitrile-acetone, in which direct measurement of the dendrite tip radius is possible [32], can only be undercooled by very small amounts so that the predicted undercooling range in which a local minimum might be observed is not accessible. In metallic systems only an indirect estimate of the tip radius is possible, generally by assuming that some characteristic microstructural length-scale, such as the grain size or dendrite trunk radius, where observable, scales as a constant multiple of the tip radius. However, although there is plentiful evidence of an initial decrease in microstructural length scale in 
the low undercooling region, it has proved almost impossible to make a continuous extension of such an analysis into the high undercooling regime where a local minimum might be inferred.

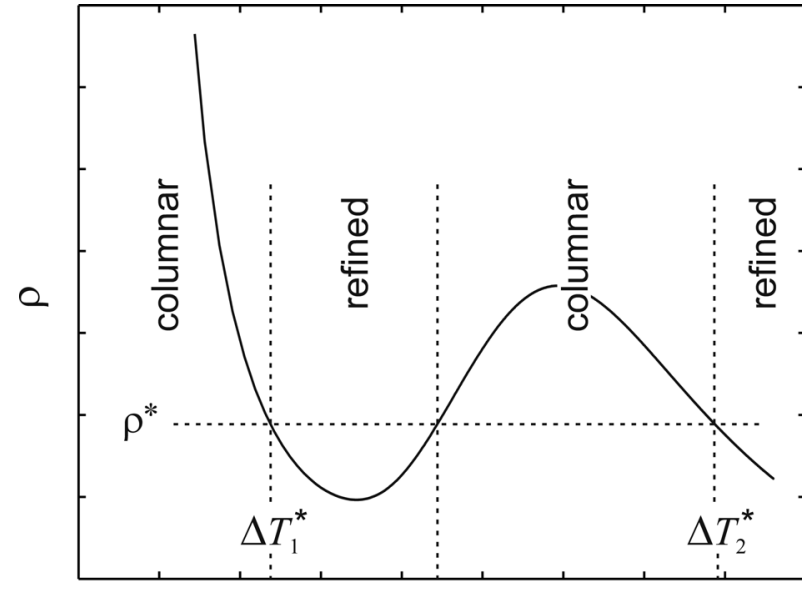

$\Delta T$
Figure 1. Schematic representation of the radius of a dendrite growing under coupled thermosolutal control as predicted by marginal stability theory and how this related to the Schwarz model for grain refinement. It is assumed that for a particular macroscopic heat extraction rate there will be a critical radius, below which remelting of the dendrite will occur. If the form of the radius curve obtained from marginal stability is correct this would lead to two grain refinement transitions.

Recently though it has become possible to use quantitative phase-field modelling to simulate the growth of dendrites under coupled thermo-solutal control [33,34], breaking the previous reliance on marginal stability models for estimating the tip radius in alloy systems where growth is sufficiently rapid that the isothermal approximation is no longer valid. Such coupled thermo-solutal simulations are extremely computationally challenging due to the severe multi-scale nature of the problem and it is only with the application of advanced numerical techniques such as adaptive meshing, implicit timestepping and efficient multigrid solvers [35] that investigations into dendritic growth in undercooled alloy systems have become feasible over significant regions of the available parameters space, including at high Lewis number [36] (Lewis number, $\mathrm{Le}$, is the ration of the thermal to solutal diffusivity, $\alpha / D$ ). In fact, such studies appear to indicate that, although the occurrence of a local minimum in $\rho$ as the undercooling is increased is indeed ubiquitous in the solidification of alloy systems, as predicted by marginal stability, a subsequent local maximum in $\rho$ at yet higher undercooling is not [37]. In fact, in a systematic study of the calculated radius-undercooling behaviour of alloy systems as a function of alloy concentration, partitioning strength and Lewis number [38] were unable to identify any part of the studied parameter space in which a maximum in $\rho$ could be observed as the undercooling was increased.

These calculations of the dendrite tip radius in undercooled alloy systems present a potentially serious problem for the accepted model of spontaneous grain refinement in alloy systems. If the radius does not display a local maximum as the undercooling is increased, then it is difficult to reconcile how a break-up time that scales monotonically with tip radius can predict two region of grain refined microstructure, nor indeed why the growth of grain refined structures above $\Delta T_{1}^{*}$ should give way to columnar growth as the undercooling is increased. However, these calculations also revealed that the tip selection parameter, $\sigma^{*}$, did show this pattern of local minimum followed by local maximum as the undercooling were increased. In fact, qualitatively, the similarities between the behaviour of $\sigma^{*}$ as predicted by the phase-field model and the form of the curve shown in Fig. 1 are remarkable. In this paper we explore whether this behaviour observed in $\sigma^{*}$ is related to spontaneous grain refinement.

\section{Description of the Model}

The model adopted here is based upon that of [33] in which, following non-dimensionalization against characteristic length and time scales, $W_{0}$ and $\tau_{0}$, the evolution of the phase-field, $\phi$, and the dimensionless concentration and temperature fields $U$ and $\theta$ are given by 


$$
\begin{gathered}
A^{2}(\psi)\left[\frac{1}{L e}+M c_{\infty}\left[1+\left(1-k_{E}\right) U\right]\right] \frac{\partial \phi}{\partial t}=\nabla \cdot\left(A^{2}(\psi) \nabla \phi\right)+\phi\left(1-\phi^{2}\right) \\
-\lambda\left(1-\phi^{2}\right)^{2}\left(\theta+M c_{\infty} U\right)-\frac{\partial}{\partial x}\left(A(\psi) A^{\prime}(\psi) \frac{\partial \phi}{\partial y}\right)+\frac{\partial}{\partial y}\left(A(\psi) A^{\prime}(\psi) \frac{\partial \phi}{\partial x}\right) \\
\left(\frac{1+k_{E}}{2}-\frac{1-k_{E}}{2} \phi\right) \frac{\partial U}{\partial t}=\nabla \cdot\left(D \frac{1-\phi}{2} \nabla U+\frac{1}{2 \sqrt{2}}\left|1+\left(1-k_{E}\right) U\right| \frac{\partial \phi}{\partial t} \frac{\nabla \phi}{\nabla \phi \mid}\right)+\frac{1}{2}\left(\left|1+\left(1-k_{E}\right) U\right| \frac{\partial \phi}{\partial t}\right) \\
\frac{\partial \theta}{\partial t}=\alpha \nabla^{2} \theta+\frac{1}{2} \frac{\partial \phi}{\partial t}
\end{gathered}
$$

where, for 4-fold growth, $A(\psi)=1+\varepsilon \cdot \cos (4 \psi), d_{0}$ is the chemical capillary length, $k_{E}$ is the partition coefficient $L$ and $c_{p}$ are the latent and specific heats respectively and $\lambda$ is a coupling parameter given by $\lambda=D / a_{2}=a_{1} W_{0} / d_{0}$ with $a_{1}$ and $a_{2}$ taking the values $5 \sqrt{ } 2 / 8$ and 0.6267 respectively [39]. $U$ and $\theta$ are related to physical concentration, $c$, and temperature, $T$, via

$$
U=\frac{1}{1-k_{E}}\left(\left(\frac{2 c / c_{\infty}}{1+k_{E}-\left(1-k_{E}\right) \phi}\right)-1\right) \text { and } \theta=\frac{\Delta T-m c_{\infty}}{L / c_{p}}
$$

where $m$ is the slope of the liquidus line, which has dimensionless form

$$
M=\frac{|m|\left(1-k_{E}\right)}{L / c_{p}}
$$

The governing equations are descritized using a finite difference approximation based upon a quadrilateral, non-uniform, locally-refined mesh with equal grid spacing in both directions. This allows the application of standard second order central difference stencils for the calculation of first and second differentials, while a compact 9-point scheme has been used for Laplacian terms, in order to reduce the mesh induced [40] anisotropy. To ensure sufficient mesh resolution around the interface region and to handle the extreme multi-scale nature of the problem at high Lewis number local mesh refinement (coarsening) is employed when the weighted sum of the gradients of $\phi, U$ and $\theta$ exceeds (falls below) some predefined value.

It has been shown elsewhere that if explicit temporal descretization schemes are used for this problem the maximum stable time-step is given by $\Delta t \leq C h^{2}$, where $C=C(\lambda, L e, \Delta T)$, with $C$ varying from $\approx 0.3$ at $L e=1$ to $C \leq 0.001$ at $L e=500$ [41], leading to unfeasibly small time-steps at high Lewis number. Consequently, an implicit temporal descretization is employed here based on the second order Backward Difference Formula with variable time-step.

When using implicit time discretisation methods it is necessary to solve a very large, but sparse, system of non-linear algebraic equations at each time-step. Multigrid methods are among the fastest available solvers for such systems and in this work we apply the non-linear generalization known as FAS (full approximation scheme [42]). The local adaptivity is accommodated via the multilevel algorithm originally proposed by Brandt [43]. The interpolation operator is bilinear while injection is used for the restriction operator. For smoothing the error we use a fully-coupled nonlinear weighted Gauss-Seidel iteration where the number of pre- and post-smoothing operations required for optimal convergence is determined empirically [41]. Full details of the numerical scheme are given in [35, 44]. 


\section{Results}

Figure 2 shows the variation of (a) the dendrite tip velocity, $V$, and (b) the equivalent parabolic [34] tip radius, $\rho$, as a function of the dimensionless undercooling, $\Delta$, for three values of the anisotropy parameter $\varepsilon$, namely $0.020,0.015$ and 0.010 . Fig. 3 shows the effective radius selection parameter, $\sigma^{*}$, calculated using the methodology described in [34]. Except for the anisotropy parameter all three sets of simulations were run under identical conditions with $\mathrm{Mc}_{\infty}=0.05, L e=200, \lambda=1$ and $\mathrm{k}_{\mathrm{E}}=0.3$. All simulations are run on a domain of $\Omega=[-1600: 1600]^{2}$ using a maximum of 12 levels of refinement, giving a minimum mesh size, $h$, of 0.78 . This is equivalent, were a uniform mesh to have been used, of a mesh size which is $2^{12} \times 2^{12}$. For each parameter set simulations were run over the undercooling range $\Delta=0.2-0.8$. Below $\Delta=0.2$ growth is very slow leading to excessive computation times while above $\Delta=0.8$ the requirement that $W_{0} V / D<<1$ is not satisfied.
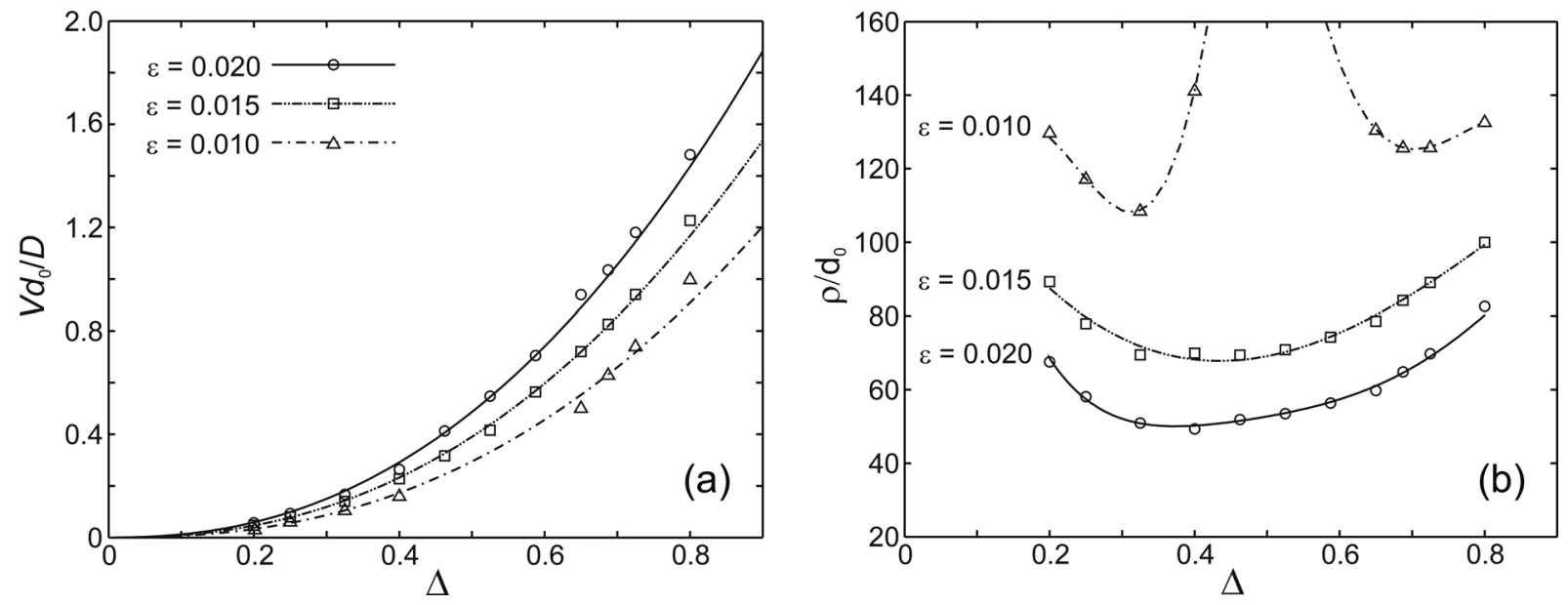

Figure 2. The dendrite growth velocity (a) and tip radius (b) as predicted by the phase-field model as a function of undercooling and anisotropy strength.

At $\varepsilon=0.020$ the results are as previously reported, with the velocity increasing monotonically with undercooling and displaying, to a very good approximation, a power-law dependence with exponent $\approx 2.3$. The radius displays a minimum at intermediate undercooling and subsequently increases at high undercooling. This results in an operating point parameter, $\sigma^{*}$, which initially decreases with increasing undercooling before passing through a local minimum to increase with undercooling. At yet higher undercooling $\sigma^{*}$ passes through a local maximum so at the highest undercoolings studied $\sigma^{*}$ is decreasing rapidly with increasing undercooling. We have previously argued [38] that this behaviour can be rationalised if the competition between solutal and thermal control of dendritic growth is manifest not in the tip radius, as for instance is evident in marginal stability models of dendrite growth [29], but in the operating point parameter, $\sigma^{*}$. We also note that in the limit $\Delta \rightarrow 0$, we find $\sigma^{*} \approx 0.07$, in good agreement with [34].

If we now consider reducing the anisotropy strength from $\varepsilon=0.020$ to $\varepsilon=0.015$ the effect on the radius and velocity is broadly in line with what we might expect. The radius follows the same trend as at the higher anisotropy level, but with a larger radius being observed at all undercoolings. This is to be expected as $\sigma^{*}$ is a monotonically decreasing function of $\varepsilon$. The velocity is correspondingly reduced, in line with the expectation that the Peclet number is only very weakly dependent upon $\varepsilon$. $\sigma^{*}$ is reduced at all undercoolings and, like the tip radius, displays the same general form as at the higher anisotropy level, although in this case we note that the difference between the minimum and maximum values is also significantly reduced. 
Finally we consider a further reduction in the anisotropy strength to $\varepsilon=0.010$, wherein a significant change in behaviour is observed. If we consider the behaviour of $\sigma^{*}$ first we observe that, in line with expectation, the value of $\sigma^{*}$ as $\Delta \rightarrow 0$ is reduced, and as with the curves for $\varepsilon=0.020$ and 0.015 , the value of $\sigma^{*}$ initially decreases as $\Delta$ is increased. However, for undercooling between $\Delta=0.4625$ and 0.5875 stable dendritic growth was not observed, with the solid nuclei used to seed solidification initially developing a preferred four-fold growth morphology but then experiencing a bifurcation at the tip which ultimately leads to a tip-splitting instability in the growing crystal. As a consequence of this tip splitting instability, in the undercooling range $\Delta=0.4625-0.5875$ neither a value for $\rho$ nor $\sigma^{*}$ could be obtained. At undercoolings above $\Delta=0.5875$ stable dendritic growth is once again established. From the values of $\sigma^{*}$ either side of this undercooling range it appears that this growth instability is consistent with a collapse to zero in the value of $\sigma^{*}$. For undercoolings either side of this unstable range the measured tip radius appears to be abnormally large, which is also consistent with the hypothesised collapse of $\sigma^{*}$, with small $\sigma^{*}$ giving rise to large values of $\rho$. In the unstable growth regime we have not attempted to determine a characteristic growth velocity as the instantaneous growth rate is subject to significant fluctuations as the morphology of the tip changes. However, once stable dendritic growth is re-established in the high undercooling regime, we note that the measured velocities are consistent with a power-law relationship with the same exponent as in the low undercooling regime.

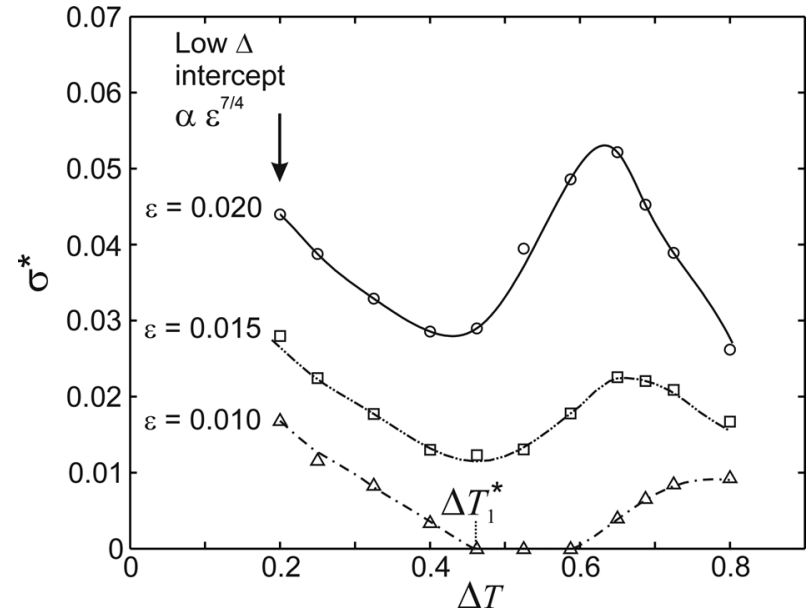

Figure 3. Calculated operating point parameter, $\sigma^{*}$, as predicted by the phase field model as a function of undercooling and anisotropy strength. At $\varepsilon=0.01$ stable dendritic growth is not observed in the undercooling range $0.4625 \leq \Delta \leq 0.5875$. Instead a tip-splitting instability leads to the growth of 'dendriticseaweed'. It is suggested that remelting of this seaweed structure gives rise to the grain-refined microstructures observed at low undercoolings.

\section{Discussion}

The breakdown of dendritic growth in phase-field simulations of solidification at high undercooling has been noted by a number of groups and has generally been attributed to a competition between capillary and kinetic anisotropies, with capillary effects dominating at low undercooling and kinetic effects dominating at high undercooling. In the case where these anisotropies are oppositely directed, doublon or dendritic seaweed morphologies, which are characteristic of growth at low anisotropy, may be observed when the competing effects are of similar magnitude. However, we do not believe that this is the case here. Firstly, the model has been constructed in the thin-interface limit, wherein the choice of parameters adopted here should eliminate all kinetics from the model and, secondly, the stable dendritic growth observed in the high undercooling regime has the same orientation as in the low undercooling regime (i.e. in both cases the dendrite arms are oriented towards the sides of the computational domain, which is the direction of the applied capillary anisotropy). This is inconsistent with an oppositely directed kinetic anisotropy which, if present, would result in growth directed towards the corners of the computational domain. By setting $\varepsilon=0$ the model has also been tested at all undercoolings considered here to determine whether there are effects resulting from mesh induced anisotropy, and this appears not to be the case. 
For the $\varepsilon=0.010$ case given in Fig. 3, the maximum in $\sigma^{*}$ observed at higher anisotropies is quite poorly developed, possibly by virtue of being shifted to somewhat higher undercooling than for the higher anisotropy simulations. Therefore, in the particular case considered here a second region of dendritic seaweed growth was not observed at higher undercoolings within the undercooling range considered. This may be an artefact of the rather low Lewis number $(L e=200)$ used in these simulations. In most metals $L e$ is typically of the order $10^{4}$ while in [38] we report that increasing the Lewis results in a more pronounced maximum in $\sigma^{*}$ and a steeper decline at high undercooling. It is currently a matter for investigation as to whether or not such simulations do result in a second region of doublon formation, although the simulations become much more computationally intensive as the Lewis number is increased due to the multi-scale nature of the problem.

\section{Summary and Conclusions}

A phase-field model of model of coupled thermo-solutal growth, constructed in the quantitative thin-interface limit has been used to study the solidification of undercooled melts over a range of undercoolings and anisotropy strengths. At moderate to high anisotropy we observe dendritic growth at all undercoolings. The tip radius initially decreases with undercooling before passing through a local minimum beyond which it subsequently increases with undercooling. However, despite studying undercoolings up to 0.8 of the hypercooling limit we found no cases in which the calculated radiusundercooling curve also displayed a local maximum. This is in distinct contrast to marginal stability theories of dendritic growth in which the radius should decrease with undercooling at very high undercooling. Consequently, if the calculated radius-undercooling curves are typical of the behaviour in metals, it is unlikely a simple decrease in the tip-radius below some limit indicative of remelting could account for spontaneous grain refinement effects in undercooled alloys. Specifically, although the calculated minimum in the radius could give rise to the low-undercooling region of grainrefinement observed in many alloys ( $\Delta T$ above $\Delta T_{1}^{*}$ in Fig. 1), it could not account for grainrefinement at high-undercooling ( $\Delta T$ above $\Delta T_{2}^{*}$ in Fig. 1), as the radius would be increasing with undercooling.

We also found that at sufficiently low anisotropy a transition of the form dendritic -> doublon/dendritic-seaweed $->$ dendritic was observed as the undercooling was increased. This can be understood by considering the behaviour of the radius selection parameter, $\sigma^{*}$, which we find to be a non-monotonic function of the undercooling. At moderate to high anisotropy, after initially decreasing with increasing undercooling, $\sigma^{*}$ displays a local minimum, beyond which it increases with further increases in undercooling until, at yet higher undercooling it displays a local maximum. Beyond this second stationary point $\sigma^{*}$ declines smoothly with undercooling. However, as the anisotropy is decreased $\sigma^{*}$ is shifted to lower values until a point is reached at which $\sigma^{*}$ collapses to zero for the intermediate undercoolings for which it had displayed a minimum. It is these regions that correspond to the observed instance of doublon or seaweed growth, rather than the growth of dendrites. We speculate that it is this transition in growth morphology that gives rise to the observed transition to a grain-refined microstructure in alloy melts at low undercooling. Noting the strong similarity between the form of the curves for $\sigma^{*}$ as a function of undercooling as calculated by the phase-field model and that for radius as a function of undercooling in marginal stability models of solidification we further speculate that grain refinement at high undercooling might be a consequence of a second region of doublon/dendritic seaweed growth resulting from a further drop in $\sigma^{*}$ at high undercooling. This has not yet been observed in the model but may exist at high Lewis number. 


\section{References}

[1] Walker J L 1959, in The Physical Chemistry of Process Metallurgy, part 2, ed. G. R. St. Pierre, Interscience

[2] Wilnecker R, Herlach D M and Feuerbacher B 1989, Phys. Rev. Lett. 622707

[3] Battersby S E, Cochrane R F and Mullis A M 1999, J. Mater. Sci. 342049

[4] Dragnevski K, Cochrane R F and Mullis A M 2004, Mater. Sci. Eng. A, 375-377 479

[5] Battersby S E, Cochrane R F and Mullis A M 2000, J. Mater. Sci. 351365

[6] Zhang T, Liu F, Wang H F and Yang G C 2010, Scripta Mater. 6343

[7] Li J F, Zhou Y H and Yang G C 2000, Mater. Sci. Eng. A 277161

[8] Li J F, Jie W Q, Yang G C and Zhou Y H 2002, Acta Mater. 501797

[9] Zhao S, Li J, Lui L \& Zhou Y H 2009, J. Crystal Growth 3111387

[10] Wang N, Gao J R, Wei B 1999, Scripta Mater. 41959

[11] Lu Y, Liu F, Yang G C, Wang H P and Zhou Y H 2007, Mater. Lett. 61987

[12] Lu S Y, Li J F and Zhou Y H 2007, J. Crystal Growth 309103

[13] Kattamis T Z and Flemings M C 1967, Mod. Casting 5297

[14] Southin R T and Weston G M 1973, J. Aust. Inst. Met. 1874

[15] Ovsiyenko D E, Maslov V V and Dneprenko V N 1979, Russ. Met. 6124

[16] Jones B L and Weston G M 1970, J. Aust. Inst. Met. 15167

[17] Jones B L and Weston G M 1970, J. Aust. Inst. Met. 15189

[18] Powell G L F and Hogan L M 1969, Trans. AIME 245407

[19] Mullis A M and Cochrane R F 1997, J. Appl. Phys. 823783

[20] Mullis A M and Cochrane R F 1998, J. Appl. Phys. 844905

[21] Schwarz M, Karma A, Eckler K and Herlach D M 1994, Phys. Rev. Lett. 731380

[22] Karma A 1998, Int. J. Non-equilibrium Processing 11201

[23] Cochrane R F, Battersby S E and Mullis A M 2001, Mater. Sci. Eng. A304-306 262

[24] Matson D 1997, in: Solidification 1998, TMS, Warrendale, PA. 233-244, ISBN 0-87339-396-1

[25] Mullis A M and Cochrane R F 2001, Acta Mater. 492205

[26] Dragnevski K, Cochrane R F and Mullis A M 2002, Phys. Rev. Lett. 89215502

[27] Bisang U \& Bilgram J H 1996, Phys. Rev. E 545309

[28] Lipton J, Glicksman M E and Kurz W 1987, Metall. Trans. A 18341

[29] Lipton J, Kurz W and Trivedi R 1987, Acta Metall. 35957

[30] Ben-Jacob E, Goldenfeld N D, Kotliar B G and Langer J S 1984, Phys. Rev. Lett. 532110

[31] Langer J S 1986, Phys. Rev. A 33435

[32] Chopra M A, Glicksman M E and Singh N B 1988, Metall. Trans. A 193087

[33] Ramirez J C, Beckermann C, Karma A and Diepers H-J 2004, Phys. Rev. E 69051607

[34] Ramirez J C and Beckermann C 2005, Acta Mater. 531721

[35] Rosam J, Jimack P and Mullis A 2007, J. Comp. Phys. 2251271

[36] Rosam J, Jimack P K and Mullis A M 2009, Phys. Rev. E 79030601

[37] Rosam J, Jimack P K and Mullis A M 2008, Acta. Mater. 564559

[38] Mullis A M 2011, Phys. Rev. E 83061601

[39] Karma A 2001, Phys. Rev. Lett. 87115701

[40] Mullis A M 2006, Comp. Mater. Sci. 36345

[41] Rosam J 2007, 'A fully implicit, fully adaptive multigrid method for multi-scale phase-field modelling', Ph.D. thesis, University of Leeds

[42] Trottenberg U, Oosterlee C and Schuller A 2001, Multigrid, Academic Press

[43] Brandt A 1977, Math. Comp. 31333

[44] Mullis A M 2010, J. Cryst. Growth 3121891 